\title{
Implementation of LTCDM -An Integrated Model for Road Networks through MobileAgents
}

\author{
T.Karthikeyan ${ }^{1}$, S.Sujatha ${ }^{2}$ \\ ${ }^{1}$ (Associate Professor, Computer Science, PSG College of Arts \& Science, India) \\ ${ }^{2}$ (Associate Professor, Computer Science, Dr.G.R.Damodaran College of Science, India)
}

\begin{abstract}
Traffic Control on roads is the major problem in modern cities. Large amount of time and resources are exhausted during traveling because of traffic congestion.Identifying emergency vehicles and providing best path is also crucial for the success of any traffic management system. This paper proposed QoS Agent and implemented an integrated Linear Traffic Control Dynamic Model (LTCDM) by integrating the mobile agents like VAISTC4 agent[6], MATLB agent[12], PCM agent[13], FMSA agent and IMAC agent[14] in order to control traffic congestion, giving prioritization of emergency vehicles ,optimizing traffic issue considering velocity of vehicles and providing best path for the vehicles on road network This linear dynamic model LTCDM also provides the better Quality of Service through the QoS Agents for road networks by improving safety applications, finding best route and driver comfort. An LTCDM is a multivariate model which uses a graph in which the vehicles are represented as nodes considering time series of flows at the various data collection site and uses finite capacity queuing theory. This dynamic \& integrated model makes real time decision making based on the host architecture and road network loads and enhancing QoS by increasing throughput and scalability on road networks with the reduction of time delay and end-to-end delay..The open source macrosimulation tool AURORA ROAD NETWORK MODELER is used for simulation to prove the significant performance of LTCDM based on the intelligent traffic \& transportation scenario.
\end{abstract}

Keywords: LTCDM, VAISTC4, FMSA, IMAC, MATLB, PCM, QoS

\section{INTRODUCTION}

Traffic Congestion is a major problem in Intelligent Transport systems on many roads worldwide. This has lead to the development of an integrated dynamic model LTCDM capable of capturing the communications between the different network components. Not only can congestion be persistent which develops due to high density of traffic seen during peak commuting hours but it also can be non persistent due to such factors as lane closures, work zones, special events etc. The severity and the location of non recurrent congestion is unpredictable. In this paper an integrated linear dynamic model LTCDM and other works in the literature discusses the use of mobile agents. Stationary or fixed gateways around the sides of roads could provide connectivity to mobile nodes (vehicles) ,but are eventually unfeasible considering the infrastructure costs involved. In such scenario, all vehicles and road side wireless devices can form a pure mobile agent adhoc network to perform vehicle to vehicle communications and achieve certain goal. In this paper nodes and vehicles are used interchangeably.

The remainder of this paper is structured as follows. In Section II, we provide a literature review of the queuing model and other mobile agents for traffic optimization and control methodologies which we consider part of the background of this paper. In Section III, we discuss The functioning of QoS Agent and Section IV gives a brief overview of LTCDM Model and its integration. Section V demonstrates how the model is implemented and its experimental results by using open source simulator tool. Finally Section VI concludes the discussion of the use of LTCDM for improving the QOS and ideas for future research in this area.

\section{LITERATURE REVIEW}

Gabriella Bretti et al[2] proposed a new simulation algorithm based on fluid dynamics that consists of numerical solutions for supporting control and solving traffic flow problems on road networks. The distinctive feature of their approach is mainly based on macroscopic fluid dynamic model. In this paper they have discussed that once congestion occurs the best effort traffic is rate controlled by calculating optimal path through a loaded network.Catriona M Queen and Casper J Albers [1] proposed a graphical dynamic model approach for traffic congestion forecasting traffic flows in road networks. The model worked in real time as required by any traffic management and intelligent system. They have also illustrated the models performance for forecasting hourly traffic flows for the particular network over a fixed time period of week days. They have proved that the model's performance may also be improved by using intervention in the parameters of the system equation. 
Mahyar Movahad Nejad et al [4] proposed two state space reduction approaches employing knowledge discovery and mathematical programming to improve the performances of the proposed approach across road network. In future they will extend the results to road networks with more general models focusing on the development of routing algorithms.R.Mynuddin Sulthani et al[10] proposed a QoS architecture for mobile adhoc networks. They have identified the messages with good delivery probability and transmitted through the path with high average node delivery index. They have also developed call admission control scheme in which calls are admitted based on the bandwidth availability of the path. Once if there is any congestion issue, the traffic rate is controlled by freeing the bandwidth for the real time flow I the existing network scheme.

In this paper, we explored an integrated dynamic model LTCDM that can automatically control the traffic congestion and enhancing the QoS by finding the best alternate route immediately for the vehicles in the existing scenario by QoS Mobile Agent.

\section{FUNCTIONING OF QOS AGENT}

The QoS Agent is the measure of a service offered by the road network to the user. The QoS Agent coordinating with the LTCDM Model is responsible for flexibility, scalability, efficiency, adaptability, reusability and maintainability.

The QoS Agent initiates the registration process by sending request to service server maintained in traffic polls. Once the request is received by services server, the registration acknowledgement is sent by the server to QoS agent. Then the Agent advertises the service to server and should be authorized by service signer. The authorized service is forwarded to parent service server which is fixed in RFID station.

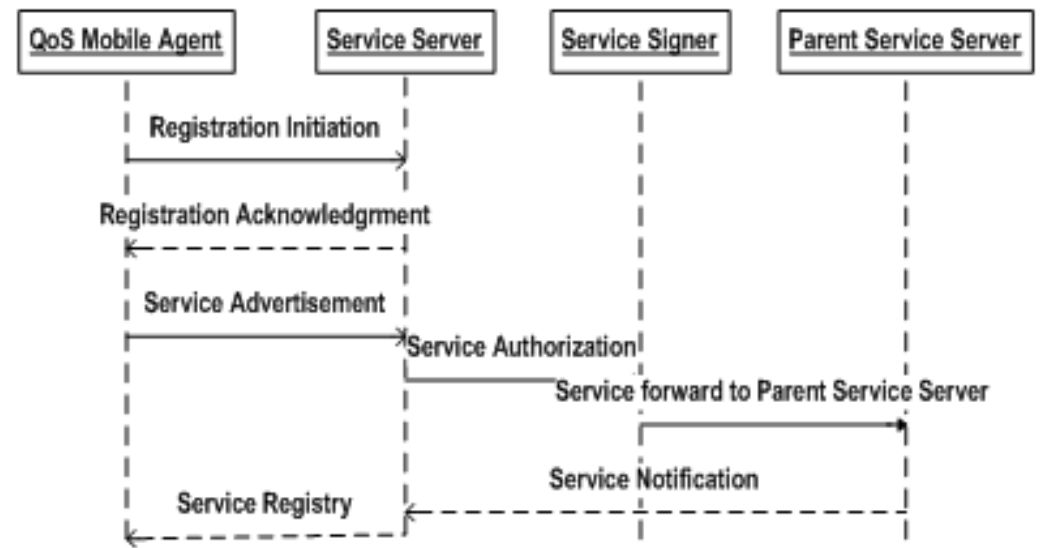

Fig 1: Registration Initiation of QoS Agent

The server in RFID Station sends a notification to child server and finally the acknowledged registration confirmation is sent to QoS agent at the first stage. At the second stage the QoS Agent sends a service request in order to locate the service in the local service server and remote service server. If there is match between the request of service sent by QoS Agent and services in local server then the locate() is invoked to provide the solution for the existing scenario on the road network. If there is no match, then the service is forwarded to the remote service server that is maintained in RFID Station and finds for the service request. If none of the service request information are matched then the QoS Agent takes real time decision for enhancing the Quality of service based on existing road network scenario.

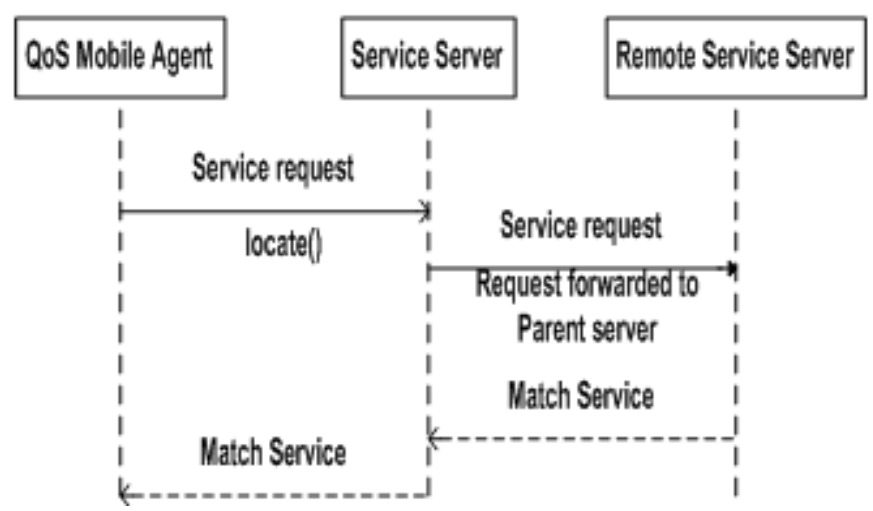

Fig 2: Service Matching of QoS Agent 
At the later stage, if the server is out of road network coverage then the remote service server acts a major role in the functioning of QoS Agent. The IMAC Agent invokes a method of connectService() to local server and it has been forwarded to Remote Service Server which is considered by service agent. If it finds better solution, then the QoS agent accepts/rejects the request and returns the result to IMAC Agent if accepted. The methods sendMessage() and receiveMessage() are invoked for the coordination between IMAC \& QoS agents and finally the connection is established to provide better solution immediately. The use of QoS Agent in LTCDM increases network resource utilization efficiency because of its adaptability to network and user requirements and exchange of minimal information during task execution and decision making with multiple resource information.

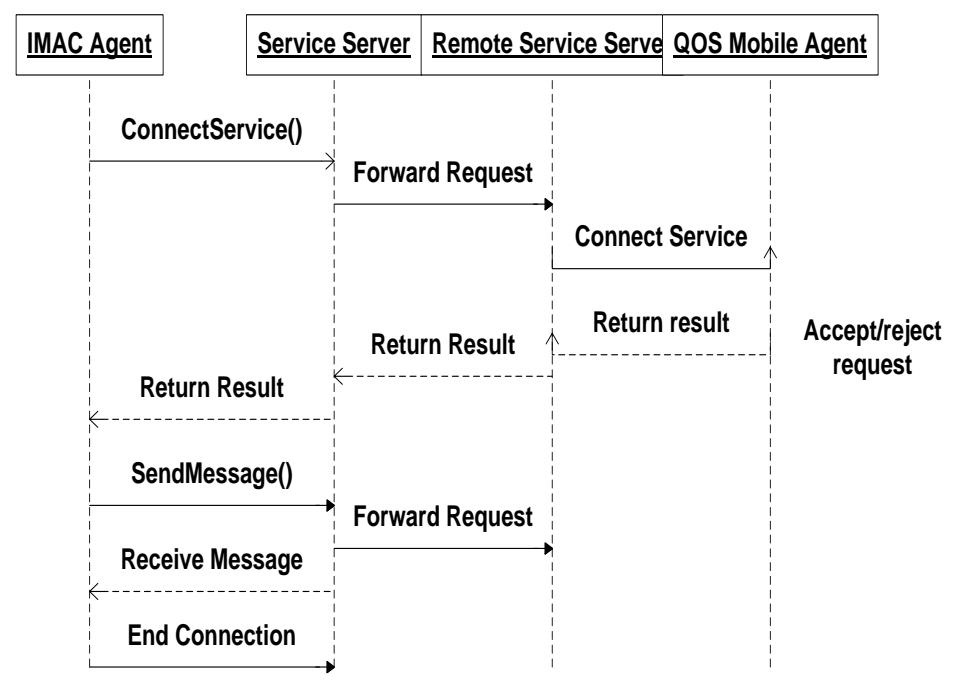

Fig 3: Connection Establishment by QoS Agent

\section{INTEGRATION\& IMPLEMENTATION OF LTCDM}

The Linear Traffic Control Dynamic Model integrates various agents like VAISTC4 (Vibrant Ambient Intelligent System for Traffic Congestion Control in Coimbatore City), MATLB (Mobile Agent for Traffic Load Balancing)[21],PCM(Prioritization and Congestion Management Agent)[13], FMSA(Fixed Monitor Stationary Agent) \& IMAC(Interactive Mobile Agent for Client) mobile agents that are supported by SALSA middleware for calculating the threshold value and also for identifying the emergency vehicles in the existing lane. This model provides better QoS to the user and in road networks by giving the real time traffic information, easy access to travel information and 24 hour real-time alternate route information[9] and better alternate route guidance for the vehicles in the lane. This LTCDM model also supports configuration of road networks ,prediction and management of QoS[10] at all the levels of abstraction (user, system and network level),management, control and performance enhancement of existing ITS.

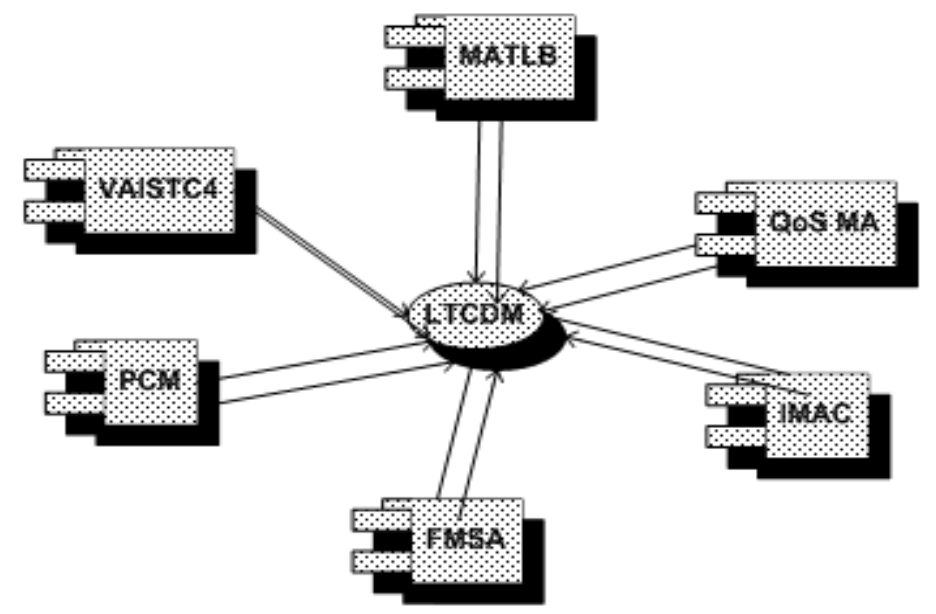

Fig 4: Component architecture of LTCDM 


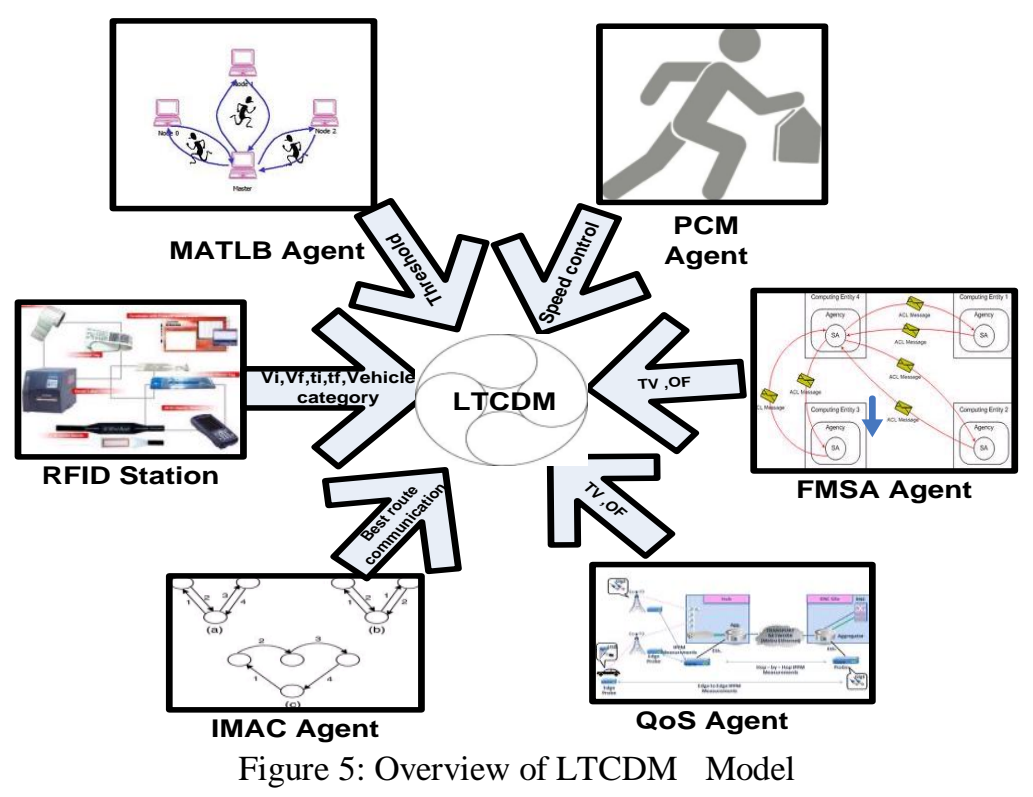

The proposed work enhances QoS in road networks by implementing LCTDM, an intelligent agent based network that can provide different messages in the form of agents.

(i) Vibrant Ambient Intelligent agent(VAISTC4)

(ii) MATLB Agent

(iii) PCM Agent

(iv) FMSA Agent

(v) IMAC Agent

(vi) QoS Agent

The core of LCTDM is creation and activation of mobile agent \& static agents and considering vehicles in the form of nodes [8].Agents are placed at intersections, RFID Stations, Traffic polls which maintain the database of traffic information and routing information except IMAC \& QoS Agents. The mobile agents in LTCDM are strategically placed such that modes are well connected and integrated with the characteristic of large buffer capacity to maintain ubiquitous database and routing table.[5]

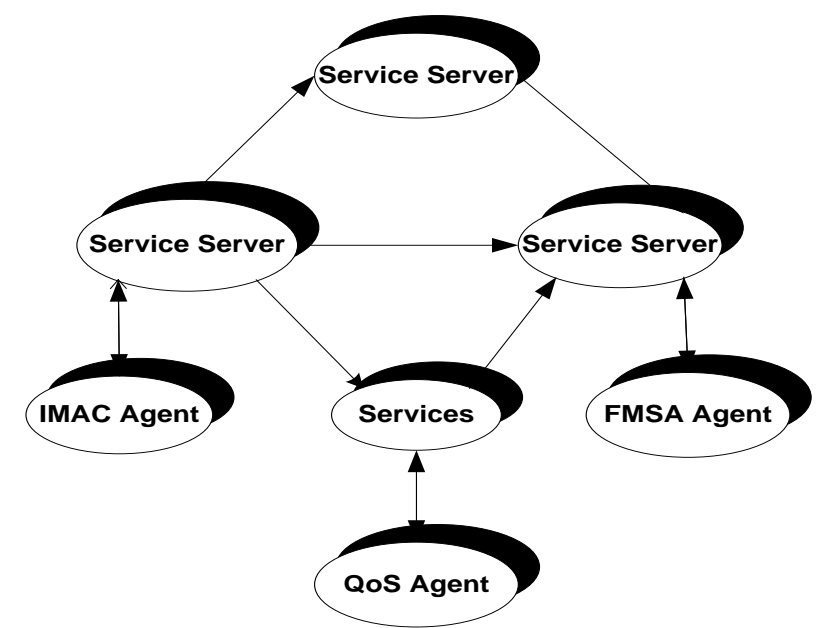

Figure6: Coordination between Mobile Agents for services

The Algorithm is executed in order to obtain best possible solution.[7]

if (node $==0$ ) then

QoS Agent is deactivated

else IMAC Agent reached to the next node then QOS Agent is activated and conveys all the information regarding path followed and resources available on that path end if 


\section{EXPERIMENTAL RESULTS}

The LTCDM proves the significant performance improvement and provides better QoS to avoid traffic congestion in great way by considering the velocity of vehicles(nodes) in the road lane.The performance of the LCTDM in co-ordination with VAISTC4 ,MATLB,PCM,FMSA,IMAC,QoS agents have been simulated on a particular day during the peak hours from $8.00 \mathrm{AM}$ to $10.00 \mathrm{AM}$ covering locations from Avinashi Road to Palladam in Coimbatore linking areas like AR(Avinashi Road), LM(Lakshmi Mills), PR(Puliakulam Road), OP(Ondipudur), LT(L\&T Bye Pass Road), SL (Sulur) and PD(Palladam) using the open source macrosimulation tool AURORA ROAD NETWORK MODELER.In the simulation, vehicles in the existing lane are considered as nodes and move with the average city speed limit of $40 \mathrm{~m} / \mathrm{s}$. Initial locations and movemnets of the nodes are obtained using the random waypoint model of AURORA simulator. It is asuumed that each in the existing lane moves independently with the same avearge speed. [11]All the agents have the same transmission link range of 250 meters.

Table 1: Simulation Settings for LTCDM

\begin{tabular}{|l|l|}
\hline No. of Nodes & $10,20,50,100 .$. \\
\hline Lane size & 1000 X 700 \\
\hline Transmission Link range & $250 \mathrm{~m}$ \\
\hline Simulation time & $50 \mathrm{~min}$ \\
\hline Traffic Source & Video \\
\hline Routing protocol & AODV \\
\hline Mobility model & Random way Point \\
\hline Node average Speed & $40 \mathrm{~m} / \mathrm{s}$ \\
\hline
\end{tabular}

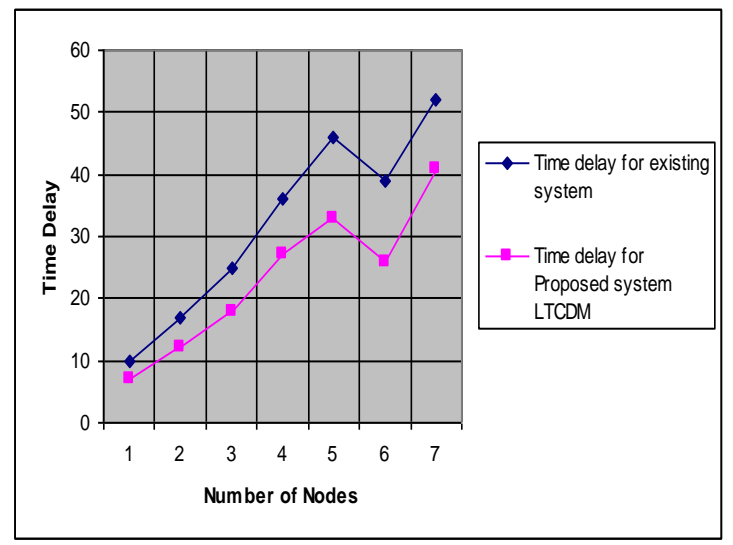

Fig 6: Simulated results for Number of nodes vs Time delay

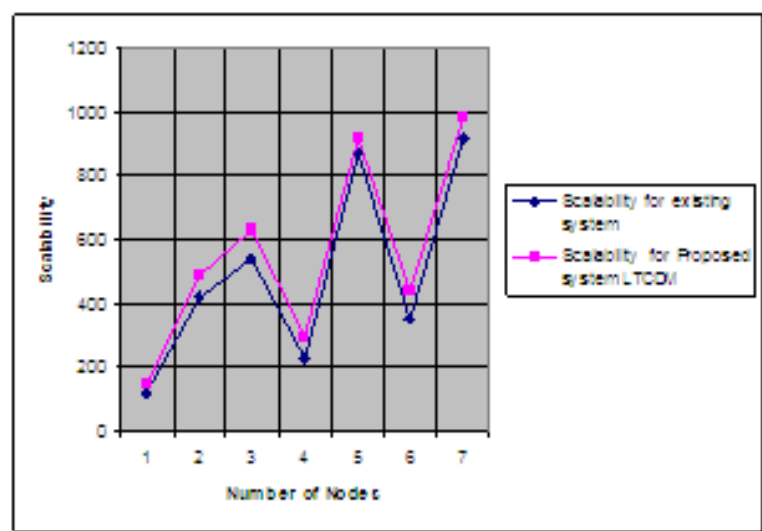

Fig \&: Simulated results for Number of nodes Vs Scalability

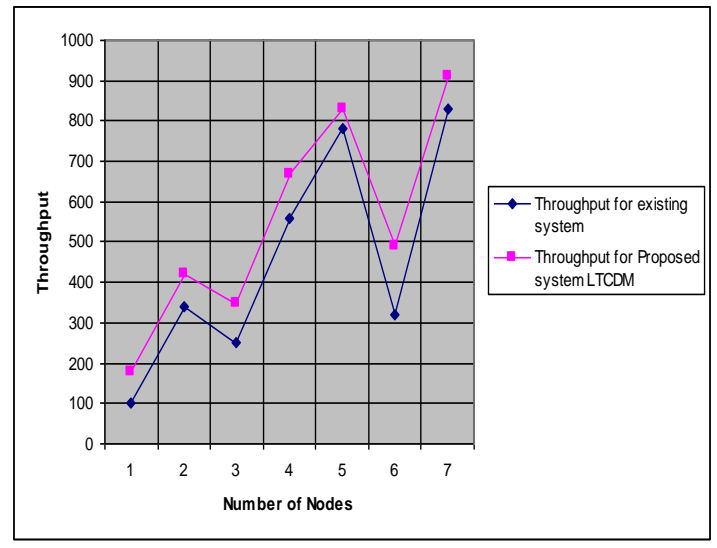

Fig 7: Simulated results for Number of nodes vs Throughput

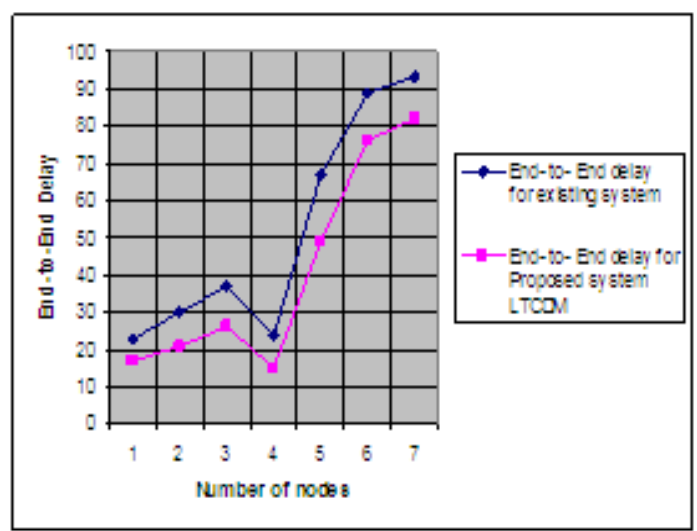

Fig 9: Simulated results for Number of nodes Vs End-to-End Delay 
From the simulation results, it is found that the LTCDM integrating mobile and stationary agents gives more throughput and Scalability, less time delay[3] and end-to-end delay when compares to the existing model. This proves that LTCDM provides the better Quality of Service through the Agents for road networks by improving safety applications, finding best route and driver comfort.

\section{CONCLUSION}

This paper focuses on the implementation of various Mobile Agents and implementation of LTCDM Model, considering real time problem in Intelligent Transportation System. The proposed LTCDM Model coordinates between various mobile agents and functioning of QoS Agent enhances the quality of service of the existing scenario by reducing congestion, decreasing time delay and end-to-end delay. The experimental study described in this paper has been carried out by AURORA open source simulator. By simulation results, we have shown that our proposed integrated dynamic model LTCDM attain high throughput and scalability with reduced time delay \& end-to-end delay compared with the existing models on road networks. Results from testing LTCDM on actual road network data from metropolitan cities are very promising. Future work may focus on mathematical approach of route guidance and for the prevention of collisions between nearby information services.

\section{REFERENCES}

[1] Catriona M Queen and Casper J Albers,Forecasting traffic flows in road Networks: A graphical dynamic model approach, Department of Mathematics and Statistics,The Open University,Milton Keynes,MK7 6AA,UK,2008.

[2] Gabriella Bretti, Roberto Natalini and Benedetto Piccoli, Fluid-Dynamic Approach to Traffic Flow problems,IACCNR,Italy.

[3] H.Jula ,M.Dessouky and P.A Ioannou, Real time estimation of travel times along the arcs and arrival times at the nodes of dynamic Stochastic networks, Intelligent Transport Systems, IEEE Transactions, Volume 9,2008.

[4] Mahyar Movahed Nejad,Lena Mashayekhy,Ali Taghavi and Ratna Babu Chinnam,State Space Reduction in Modeling Traffic Network Dynamics for Dynamic Routing Under ITS ,International IEEE Conference on Intelligent Transport Sytsems, Washington,DC,USA,2011.

[5] Naishadh K Dave and Vanaraj B Vaghela,Vehicuar Traffic Control : A Ubiquitous Computing Approach ,IC3 2009,CCIS 40,2009.

[6] N.Sudha Bhuvaneswari, S.Sujatha (2010), Vibrant Ambient Intelligent System for traffic congestion control in Coimbatore city(VAISTC4), Proceedings of the $12^{\text {th }}$ International Conference on Networking, VLSI and Signal Processing, Pg.No. 288-293

[7] Pitu Mirchandani,Larry Head,Rhodes : A Real Time Traffic Signal Control System: Architecture, Algorithms and Analysis.

[8] Sadaf Momeni,Mahmood Fathy,VANET Communication,IEEE,2008.

[9] S.Kim ,M.E.Lewis and C.C White III ,State Space reduction for non stationary stochastic shortest path problems with real time traffic information, Intelligent Transport Systems IEEE Transactions, Volume 6,2005.

[10] Sulthani,R.M.,Rao.D.S: Design of an Efficient QoS Architecture (DEQA) for Mobile Adhoc Networks.ICGST-CNIR Journal Volume 8,Issue 2,2009.

[11] Tebaldi C. West, M and Karr A.K, Statistical Analyses of freeway traffic flows, Journal of forecasting, 21,2002.

[12] T.Karthikeyan, S.Sujatha, N.Sudha Bhuvaneswari(2012), Mobile Agent Based Approach for Traffic Load Balancing using Sensors, International Journal of Computer Applications(IJCA), Volume 47, Number 6.

[13] T.Karthikeyan, N.Sudha Bhuvaneswari, , S.Sujatha (2012), Traffic Handling Approach with Intelligent Speed Control and Prioritization of Emergency Vehicles using PCM Agent ,International Journal of Computer Technology and Application(IJCTA), Volume 3,Issue 4.

[14] T.Karthikeyan,S.Sujatha(2012), Optimization of traffic System using TCL Algorithm through FMSA and IMAC Agents,International Journal of Advanced Research in Computer Engineering \& Technology(IJARCET), Voulme 1 ,Issue 6 .

\section{AUTHOR PROFILE}

Thirunavu Karthikeyan received his graduate degree in mathematics form Madras University, Post graduate degree in Applied Mathematics from Bharathidasan University and received doctoral degree in Computer Science form Bharathiar University. Presently he is working as an Associate Professor in Computer Science, PSG College of Arts \& Science, Coimbatore. His research interests include image coding, Mobile agents, Pattern Recognition etc. He has published many papers in National and International Conferences and Journals. He has completed many funded projects with excellent comments.

S.Sujatha started as a Computer Science graduate. She completed her undergraduate degree at Sri Sarada College for Women, Tirunelveli and has also completed post graduate level courses MCA and MPhil at Bharathiar University, Coimbatore, India, and is currently pursuing her doctorate in Computer Science. Her area of interest is Mobile Agent Technology \& Networks. She has been participating continuously in research and development activities for the past ten years. To her credit, she has presented and published technical papers in International Journals, at International Conferences and International Workshops organized by various international bodies like IEEE, WSEAS, and IEEE Explore. She has published book on Integrating Service Oriented Architecture and Web Services and also contributed chapters in books like Personal Area Network and published articles and working manuals in agent technology. The author is currently employed as Associate Professor at the Dr. G.R Damodaran College of Science, Coimbatore, India. She is an active member of various echnical bodies like ECMA, Internet Society of Kolkata and Chennai and acts as a moderator in various international conferences and journals. 